\title{
Neural Information Processing: between synchrony and chaos
}

\author{
Josep L. Rosselló, V. Canals and A. Morro \\ Physics Department, Universitat de les Illes Balears, Palma de Mallorca, Spain
}

\section{Summary}

The brain is characterized by performing many different processing tasks ranging from elaborate processes as pattern recognition, memory or decision-making to more simple functionalities as linear filtering in image processing. Understanding the mechanisms by which the brain is able to produce such a different range of cortical operations remains a fundamental problem in neuroscience. Some recent empirical and theoretical results support the notion that the brain is naturally poised near critically between ordered and chaotic states. As the largest number of metastable states exists at a point near the transition, the brain can therefore access to a larger repertoire of behaviours. Consequently, is of high interest to know which type of processing can be associated to both ordered and disordered states. Here we show an explanation of which processes are related to chaotic and synchronized states based on the study of in-silico implementation of biologically plausible neural systems. The measurements obtained reveals that synchronized cells (that can be understood as ordered states of the brain) are related to non-linear computations while uncorrelated neural ensembles are excellent information transmission systems that are able to implement linear transformations (as the realization of convolution products) and to parallelize neural processes. From these results we propose a plausible meaning for Hebbian and non-Hebbian learning rules as those biophysical mechanisms by which the brain creates ordered or chaotic ensembles depending on the desired functionality. The measurements that we obtain from the hardware implementation of different neural systems endorse the fact that the brain is working with two different states, ordered and chaotic, with complementary functionalities that imply a non-linear processing (synchronized states) and information transmission and convolution (chaotic states).

\section{Introduction}

Understanding the brain and to reach the ability to reproduce its amazing processing capabilities is one of the most challenging purposes for science and technology. These processing capabilities are of different nature as body control, high-speed signal processing and classification, memory, decision-making etc. Since these diverse processes depend on the neural behaviour, it is crucial to know which type of connectivity or environmental conditions is associated to each process. Recently, some researchers support the theory that the brain is naturally poised near critically ${ }^{1,2}$, at the edge between ordered and chaotic states. This duality implies that the brain can access to a large range of complementary behaviours related to these two opposite states and it could be an explanation of the diversity of brain processes.

A feasible way to study the different brain capabilities is to artificially reproduce those systems by using biologically plausible neural implementations. In this sense, hardware solutions are much more trustworthy and quicker than software approaches since the intrinsic neural parallelism is maintained. From the different hardware solutions that can be considered, those based on digital logic represents the more attractive way for studying neural systems ${ }^{3}$ since they can be massively implemented in Field Programmable Gate Arrays ${ }^{4}$ (FPGAs) where hundreds of coupled neurons can be configured in a single chip for its detailed study.

Understanding complex systems always entails choosing a level of description that retains key properties of the system while removing what is supposed to be nonessential. In this sense, spiking neural models ${ }^{5,6}$ are bio-inspired descriptions that use delta functions to represent the Action Potentials of biological neurons. Implicitly this assumes that no information is transmitted from cell to cell in the spike shape but on its timing characteristics, thus implying a temporal codification. There is no question that firing rate codes are used in nervous systems ${ }^{7}$. To what extent more complex timing codes exist remains a subject of considerable debate ${ }^{8}$. 
A key property of the spike trains measured in real neural systems is their seemingly stochastic or random nature ${ }^{9}$. In fact, the apparent lack of reproducible spike patterns has been one of the principal arguments in favour of firing rate coding. The stochastic nature of spike trains is in part due to the mechanism of synaptic transmission since each synaptic vesicle releases its "quantum" of transmitter from the neuron presynaptic terminal with a given probability. This probability of synaptic release is subject to change and can be understood as a measurement of the connection weight. Due to this probabilistic nature a basic feature of neuronal communication is the lack of transmission reliability ${ }^{8,9}$. Moreover, as the number of channels is reduced, the axonal membrane can spontaneously produce action potentials, ${ }^{10}$ thus generating an autonomous internal activity.

The chaotic fluctuation of the resting potential of neurons ${ }^{9}$ can provide a possible explanation for the different processing tasks that are performed by the brain. A synchrony (asynchrony) in the activity should create correlated (uncorrelated) random oscillations. The interaction of those random oscillations (between correlated or uncorrelated variables) would lead to different behaviours.

In this paper we show a simple stochastic neural model based on digital circuitry that implements the basic Leaky Integrate-and-Fire neural model and also reproduces the probabilistic nature of synaptic transmissions. This is, to the best of our knowledge, the first time in which a stochastic neural model is implemented digitally. From this model we study the different processing capabilities that can arise in neural systems, showing that neuron ensembles present very different properties depending on their relationship.

\section{The role of synchronism and chaotic behaviour in neural processing}

One of the key points of the stochastic neural model selected is that the difference between the threshold and the resting potentials is variable and can synchronize neurons (see the methods section). Note that synchronicity does not necessary implies a common periodic oscillation, and any kind of coupled oscillation would provide similar results.

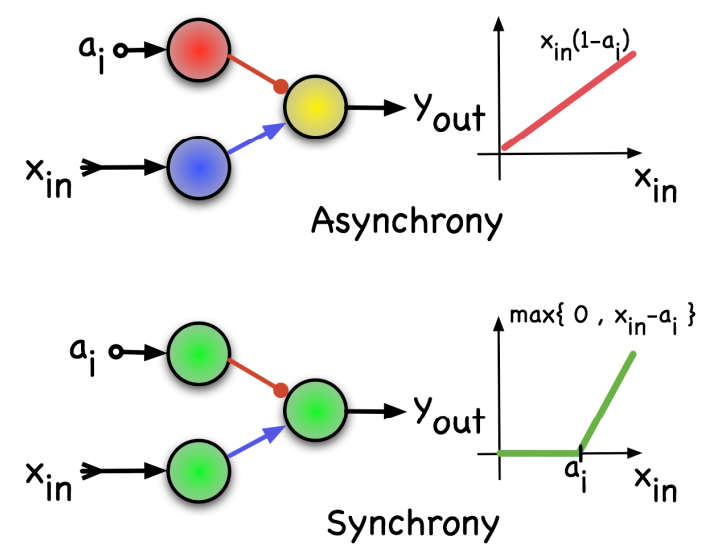

(a)

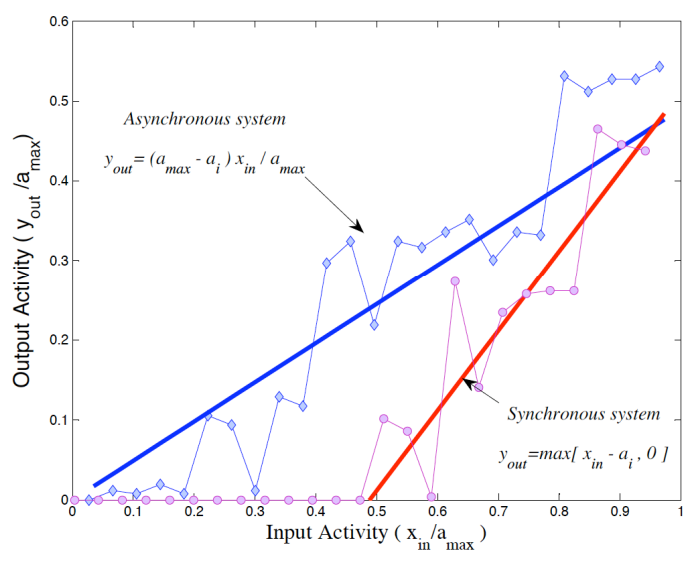

(b)

Fig. 1. Basic measured differences between asynchronous and synchronous neural systems: (a) Simple example of synchronous and asynchronous systems. (b) Measured output activities for the two cases when using the digital stochastic neural model.

To highlight the differences that can be observed we show the simple example of Fig. 1a. This figure illustrates two simple systems consisting in two input neurons with an excitatory and an inhibitory connection with an output neuron. One of the two input activities is fixed $\left(a_{i}\right)$ while the other is varied $\left(x_{i n}\right)$. In the absence of synchrony between neurons we observe a nearly linear relationship between the output activity $y_{\text {out }}$ and the two input activities $\left(a_{i}\right.$ and $\left.x_{i n}\right)$. For the case of synchronized neurons (identical colours indicate synchronization) we observe that when the input $x_{i n}$ is lower than the inhibition activity $\left(a_{i}\right)$ the excitatory signal is unable to activate the output since it is correlated with the inhibitory signal. Once the excitation exceeds the inhibition we get an appreciable output activity that can be 
approximated as being linear $\left(y_{\text {out }}=x_{i n}-a_{i}\right)$. In Fig. $1 \mathrm{~b}$ we show the measurements taken for these systems by using the proposed digital neural model.

Therefore, in the synchronous case we observe an abrupt non-linear change between an OFF (for $x_{i n}<a_{i}$ ) and an ON state that is not present in the uncorrelated case (where an activity at the output is always appreciable for any value of $x_{i n}$ ). The oscillatory values measured in Fig. $1 \mathrm{~b}$ around the expected behaviour are due to the intrinsic stochastic activity of neurons, where the firing rates are measured averaging in a limited time period.

Therefore, the first and crucial observation that is derived from the digital stochastic neural model is that uncorrelated and correlated neural systems present a linear and a non-linear behaviour respectively. In case of synchrony we observe that the system selects its operation mode between different linear states while in the case of a chaotic behaviour we observe a simple linear transformation.

\section{Chaotic ensembles of neurons as efficient information processing systems}

As has been suggested, ensembles of neurons that are not synchronized seem to perform linear transformations to neural information. Uncorrelated systems can therefore be used to implement the convolution product to neural signals. One of the well-known properties of simple cells in the mammalian visual cortex is their ability to implement $2 \mathrm{D}$ linear filters to input visual objects ${ }^{11,12}$. It is found that neural circuitry in the mammalian visual cortex is able to respond to images in a given orientation while filtering the others. This is due to the ability of simple receptive fields of implementing 2D Gabor filters. The main supposition is that this filtering is the first step of a series of neural actions committed to recognize objects and shapes.

A Gabor filter can be implemented using a simple feed-forward network with only two layers of neurons (see Fig.2a). The input image creates a neural activity proportional to the light intensity that stimulates the output layer. The weights of the connections (that is represented by the probability of stimulus transmission) follows a Gabor-like shape with an overall function of implementing the convolution of the image with the unit impulse response of the Gabor filter.

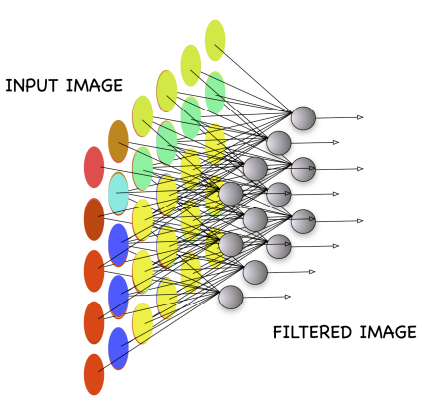

(a)

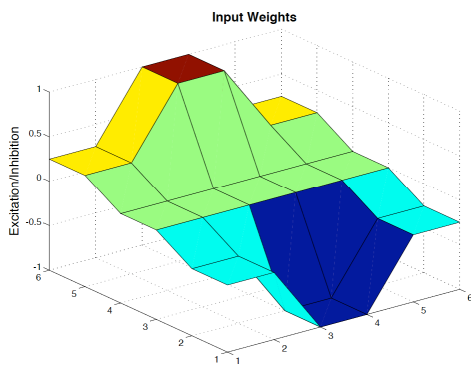

(b)
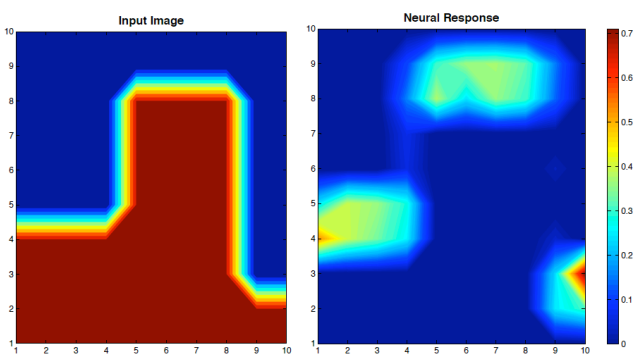

(c)

Fig. 2. a) Simple Feed-forward neural system used for image filtering. b) Weights used for the connection between the input and the output layer. Positive (negative) values represent excitatory (inhibitory) connections. c) Neural response (right image) of the feed-forward stochastic spiking neural network to an input image (left).

The main functionality of this Gabor-like distribution is to enhance the edges of the image in a pre-defined orientation while attenuating any other characteristic.

To prove the capability of uncorrelated neural circuits to implement linear filters (in this case a 2D Gabor filtering) we created a simple neural network with one output layer of 100 neurons that are processing an image of $10 \times 10$ pixels. Each output neuron of the network is connected to nine inputs of the image (pixels) with the weight distribution shown in Fig. 2b. In Fig.2c we show the input image to be filtered and the activity induced at the output layer of the network. As can be appreciated, the horizontal edges are highlighted while the other 
features are filtered. Using similar methodologies any kind of linear filtering (as a complete edge detection system) can be built using a simple feed-forward neural network. Due to the low number of layers used (in this case 1 single layer) the computation speed is quite high since only one single synapse is needed to filter the image. For the implementation of the digital circuit we used an ALTERA Cyclone III FPGA and obtained a computation speed of about 40ns. This high computation speed is due to the intrinsic parallelism of neural systems and enables its use for ultra high-speed signal processing.

\section{Synchronized systems are able to recognize patterns while chaos improves and speeds-up the process}

Pattern recognition is a fundamental brain process that consists in a reaction of the system when an external stimulus is delimited in a given range. Some works suggest that this process is explained using the action potential timing as codification methodology ${ }^{13}$ but this mechanism requires great demands on the spatiotemporal precision with which neurons need to be wired up and the learning process of such coding would be quite complex. Some studies have demonstrated that visual pattern analysis and pattern classification carried out by macaque monkeys can complete its computation in just $20-30 \mathrm{~ms} .{ }^{14,15}$ Since the firing rate of neurons are usually below $100 \mathrm{~Hz}$, a coding of analogue variables by firing rates is traditionally considered to be dubious for fast cortical computations. Other studies demonstrated that when odorevoked action potentials in honeybee antennal lobe neurons are pharmacologically desynchronized (without interrupting neural activity) fine olfactory discrimination is interrupted ${ }^{16,17}$. This is an evidence linking directly neuronal synchronization to pattern recognition. Here we suggest that synchronized systems (that presents the ability of switching between ON and OFF states), are responsible of the pattern recognition process by using a firing rate coding. The proposed mechanism only needs a low number of synaptic steps (three basic steps) that ensure a fast recognition process (in agreement with the in-vivo measurements ${ }^{14,15}$ ).

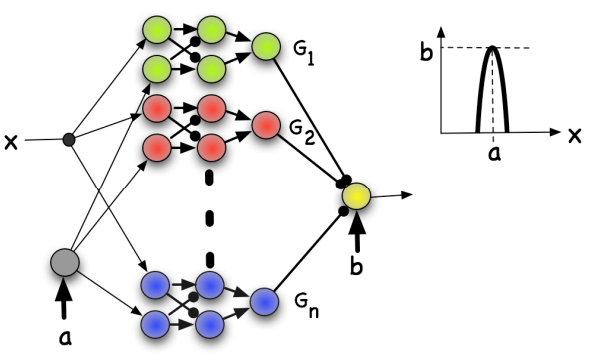

(a)

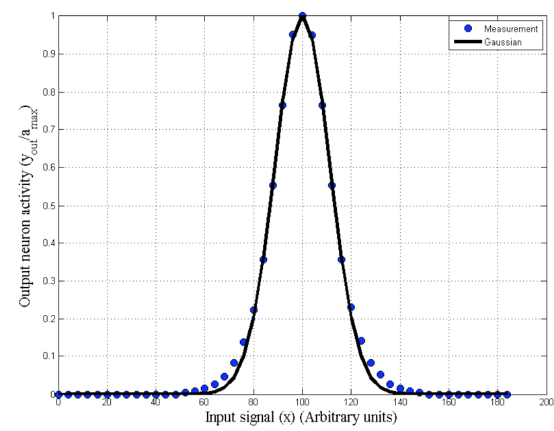

(b)

Fig. 3. a) A simple circuit scheme that is able to recognize. b) Measured activity of the proposed system when $n=32$

In Fig. 3a we show a simple scheme where different synchronized neuron ensembles $\left(G_{1}, G_{2}, . ., G_{n}\right)$ are recognizing the point in which the input stimuli $x$ is near a reference activity $a$. The output activity of each synchronized ensemble vanishes only when $x=a$, thus allowing the output neuron to reach its maximum activity (b). If the stimuli $(x)$ is not in the neighbourhood of $a$ then all the ensembles (that are not synchronized between them) inhibits the activity of the output neuron. The result is a Gaussian-like response of the output with respect the input (see Fig. 3b). Note the differences in signal fluctuation between this figure and Fig $1 \mathrm{~b}$ due to the difference in systems sizes ( 3 neuron vs. more than one hundred). The system response is quite fast since only a few synaptic steps are involved. The dispersion of the Gaussian function depends on the number of synchronized ensembles (the sigma value decreases as $n$ increases). Measurements of its timing activity is shown in Fig. 4a where we represent the neural behaviour before (input=0) and after (input $=1$ ) the sensed stimuli is within the range of 
detection. Before the detection the synchronized ensembles presents an appreciable activity and the output neuron is unable to switch. After the transition the ensembles activity practically vanishes and the output neuron immediately starts to fire (just indicating the presence of the desired pattern).
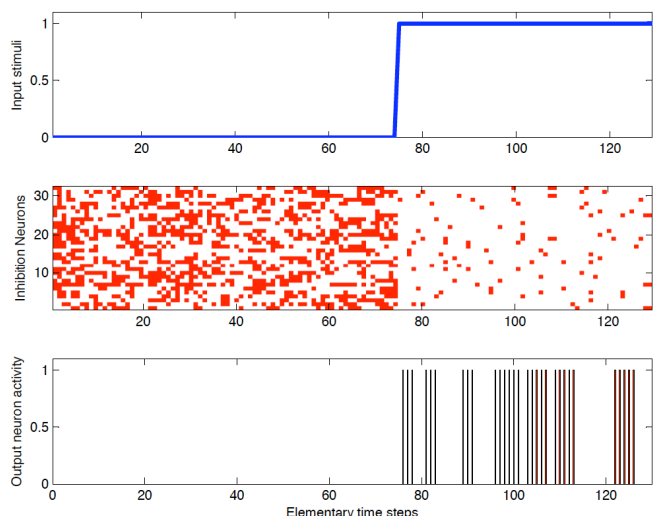

(a)

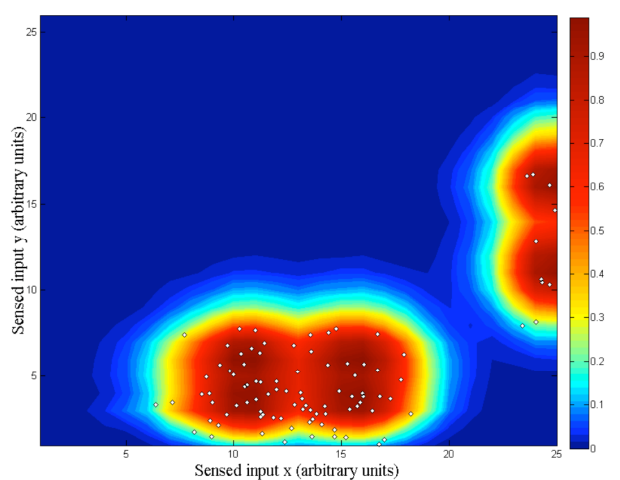

(b)

Fig. 4. a) Evaluation of the time response of the pattern recognition system, a low number of synaptic transmissions is needed to recognize

the signal. b) Generalization to two-dimensional non-gaussian pattern recognition. The system is able to correctly recognize the class

(symbols) scattered in a non-gaussian distribution.

The circuit of Fig.3a (adapted only for 1D signals and Gaussian discriminations) is easily generalized to higher dimensions and non-gaussian patterns by superposing different Gaussian (kernels) functions. An example of non-gaussian pattern recognition is shown in Fig. $4 \mathrm{~b}$, where we represent the activity of the output neuron of a classifier (the colour map indicate this activity) as a function on the values of two sensed signals $(x$ and $y$ ). The non-guassian discrimination has been obtained by superposing different Gaussian kernels, thus obtaining a Parzen classifier that is a good approximation to Bayesian classifiers. To train the network we only require some reference points of the desired signals to be recognized (in this case different vectors $\boldsymbol{a}_{i}$ ) at the centre of each Gaussian kernel (to obtain the results of Fig. $4 \mathrm{~b}$ we used 6 reference vectors). The advantage of this neural classifier is that the training of the network is not performed in a complex way (as the use of back-propagating algorithms that need to be used in traditional Artificial Neural Networks) since the same sensed signal can be used for the training. That is, the system can be self-configured easily since the sensed stimuli can be incorporated to the network as a reference signal. The only requirement is that other parts of the brain must fix those vectors of interest when some catastrophic phenomena arise (and therefore every time the signals are within the memorized range the phenomena is evoked).

The circuit shown in Fig. 3a is only a simple example of how a Bayesian classifier can be implemented with synchronized systems. Probably there are many other configurations that can implement a similar computation but the concept is that, pattern classifiers with a few synaptic connections and with a simple training methodology (in this case the measurement is the training) can be implemented by using synchronized neural ensembles. Note that in Fig. 3a the different neural ensembles are not synchronized between them since they must completely inhibit the output neuron (a pure synchronized system would fail in a complete inhibition of the output neuron when the stimuli is out of the desired range of detection). The result is an improvement of the neural comparison by parallelizing the process due to the independence between the synchronized neural clusters. 


\section{Hebbian and non-Hebbian learning rules understood as the methodologies used to select between}

\section{opposite functionalities}

In the light of the previous results we postulate that both Hebbian and non-Hebbian learning rules can be understood as the biophysical mechanisms that are responsible of creating ensembles of correlated and un-correlated neural systems. Hebbian learning rule states that the simultaneous activation of cells leads to pronounced increases in the synaptic strength between those cells. That is, neurons that fire together wire together but no matter if this common firing is due to the same external signal or not (this is the case of the system of Fig. 1a where two independent signals $a_{i}$ and $x_{i n}$ converge to the same synchronous neural circuit). Therefore, we can associate Hebbian and non-Hebbian learning rules to the implementation of completely different functional systems: non-linear and linear processes respectively (see Fig. 5).

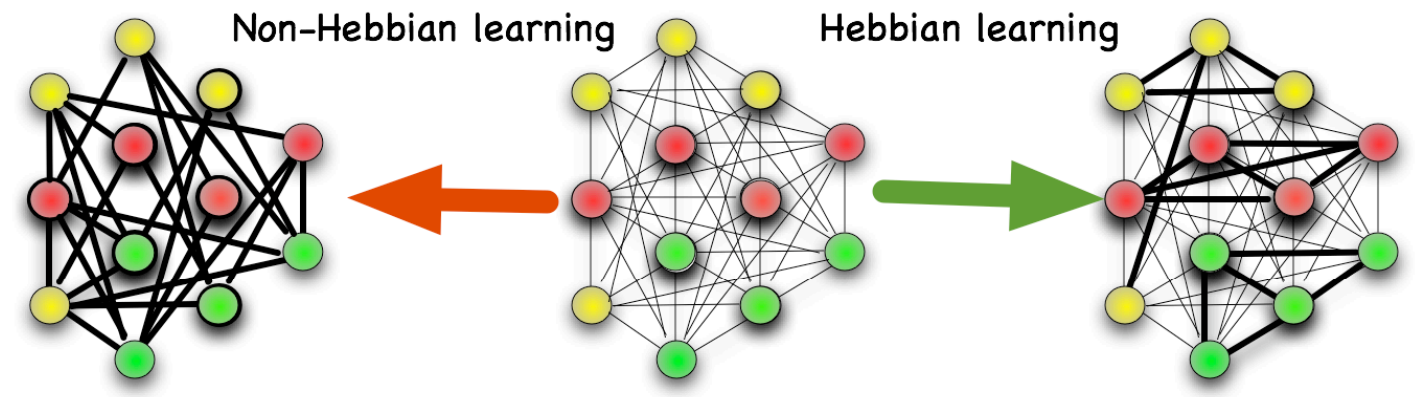

\section{Network operating in the} linear regime

Network operating in the non-linear regime

Fig. 5. Hebbian and non-Hebbian learning rules can be understood as the bio-physical mechanisms necessary to implement non-linear and linear processes respectively

Methods: Implementing and measuring stochastic neural systems

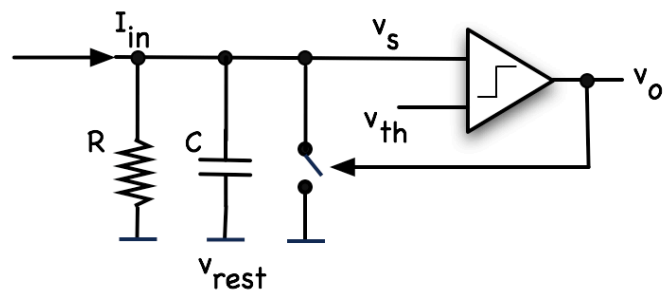

(a)

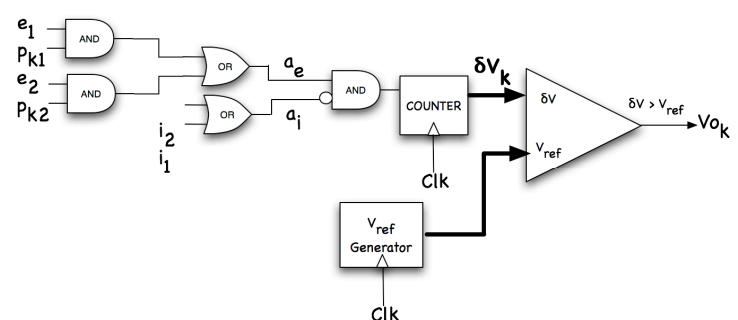

(b)

Fig. 6. a) The Leaky Integrate-and-Fire neural model. b) Digital implementation of the Leaky Integrate-and-Fire neural model

The model that we use for neural implementation is the Leaky Integrate and Fire (IF) (see Fig. 6a). This model is widely used for neural description and can be easily implemented using digital circuitry. Each neuron has an incoming current created by the spike trains of the different synapses of the neuron (input current $I_{i n}$ in Fig. 6a). This current increases the membrane potential over the resting potential ( $v_{s}$ and $v_{\text {rest }}$ respectively) and an output spike is generated when the membrane potential is over a threshold value $\left(v_{t h}\right)$. After the spike generation, the membrane potential returns to the resting state and $v_{s}=v_{\text {rest }}$. From Ohm's law, the membrane potential in the stationary state can provide us an idea of the relationship between $v_{s}$ and $I_{\text {in }}\left(v_{s}=v_{\text {rest }}+I_{\text {in }} R\right)$. Therefore, if and only if the membrane over-voltage $\left(\delta V_{s} \equiv v_{s}-v_{\text {rest }}=I_{\text {in }} R\right)$ is greater than $v_{t h}-v_{\text {rest }}$, an action potential at $v_{o}$ is generated. 
The simple mechanism of the IF neural model can be reproduced digitally as shown in Fig. 6b (example for a two-input neuron scheme that can be generalized to the multiple input case). All the incoming excitatory signals $\left(e_{i}\right)$ are joined using an OR gate and are increasing the value of a digital block (the counter) that provides at its output the number of incoming pulses during a certain period of time (an estimation of the incoming current and therefore the membrane overvoltage). At the same time, shunting inhibition signals ( $\left.i_{j}\right)$ inhibits the action of excitatory pulses. Signals $\mathrm{p}_{\mathrm{kj}}$ represent the probability of signal transmission from the $j_{t h}$ to the $k_{t h}$ neuron and are binary signals oscillating with a given probability $\left(p_{k j}\right)$ generated using Linear-Feedback Shift Registers (LFSRs) or with a chaos-based random number generator ${ }^{18,19}$. Therefore, $p_{k j}$ measures the strength of connection between the two neurons and its biophysical meaning is related to the probability of vesicle release in the synaptic connection.

The approximated value of membrane overvoltage $\left(\delta V_{s}\right)$ is then compared with a reference signal ( $\left.v_{\text {ref }}\right)$ representing the difference between the threshold and the resting voltage $\left(v_{r e f}=v_{t h}-v_{\text {rest }}\right)$. This reference signal is generated using a random signal generator between a maximum and a minimum value. The digital circuit used in this work were implemented using a Field-Programmable Gate Array from ALTERA (a Cyclone III EP3C16F484C6).

\section{Discussion}

Using a new digital stochastic neural model we implemented different neural systems incorporating hundreds of neurons for its study. The measurements show that neurons are unique processing elements that are able to change its functionality drastically depending on the correlations among spike trains. Simple processes as image convolution are perfectly implemented by chaotic systems. In addition, complex processes as pattern recognition or decision making can be associated to synchronized groups of neurons since those systems are able to "chose" between different states. At the same time, a chaotic relationship between synchronized systems seems to improve the complex processes by parallelizing them (and thus speeding up de process). As a consequence of that, it has been demonstrated that firing rate codes cannot be discarded to be involved in the pattern recognition process since the proposed mixed system (with both chaotic and ordered neural ensembles) is able to recognize patterns using a low number of synaptic steps, thus explaining the fast pattern recognition process observed in the mammalian cortex. Finally we provide a new meaning for the Hebbian and non-Hebbian learning rules as the mechanisms that are able to select between opposite functionalities.

\section{References}

1. Bertschinger, N., Natschläger, T. Real-Time Computation at the Edge of Chaos in Recurrent Neural Networks. Neural Computation 16 (7), 1413-1436 (2004)

2. Chialvo, D. Emergent complex neural dynamics. Nature Physics 6 744-750 (2010)

3. Hishiki, T., Torikai, H. A novel rotate-and-fire digital spiking neuron and its neuron-like bifurcations and responses. IEEE Transactions on Neural Networks 22 (5), 752-767 (2011)

4. Maeda, Y., Tada, T. FPGA implementation of a pulse density neural network with learning ability using simultaneous perturbation. IEEE Transactions on Neural Networks 14 (3), 688-695 (2003)

5. Maass, W. Networks of spiking neurons: The third generation of neural network models. Neural Networks 10, (9) (1997) pp. 1659-1671

6. Ghosh-Dastidar, S., Adeli, H., Spiking Neural Networks, International Journal of Neural Systems, 19 (4) (2009) pp. 295-308

7. London, M., Roth, A., Beeren, L., Häusser, M., Latham, P.E. Sensitivity to perturbations in vivo implies high noise and suggests rate coding in cortex. Nature 466 (7302) 123-127 (2010) 
8. Koch. C. Biophysics of Computation, Information Processing in Single Neurons, (Oxford University Press, New York, 1999)

9. Steinmetz, P.N., Manwani, A., Koch, C., London, M., Segev, I. Subthreshold voltage noise due to channel fluctuations in active neuronal membranes. Journal of Computational Neuroscience. 9 (2) 133-148

10. Chow, C. C. and White, J. A. Spontaneous action potentials due to channel fluctuations. Biophysical Journal 71 (6) $3013-3021$ (1996)

11. Daugman, J.G. Uncertainty relation for resolution in space, spatial frequency, and orientation optimized by two-dimensional visual cortical filters, J Opt. Soc. Am. 2 (7) 1160-1169 (1985)

12. Jones, J.P., Palmer, L.A. An evaluation of the two-dimensional gabor filter of simple receptive fields in cat striate cortex, $J$. Neurophysiology. 58 (6) 1233-1258 (1987)

13. Hopfield J.J. Pattern recognition computation using action potential timing for stimulus representation. Nature 376 33-36 (1995)

14. Rolls, E.T. Brain mechanisms for invariant visual recognition and learning. Behavioural Processes 33 (1-2) 113-138 (1994)

15. Rolls, E.T., Tovee, M.J. Processing speed in the cerebral cortex and the neurophysiology of visual masking. Proc. of the Royal Society B: Biological Sciences 257 (1348) 9-15 (1994)

16. Stopfer, M., Bhagavan, S., Smith, B.H., Laurent, G. Impaired odour discrimination on desynchronization of odour-encoding neural assemblies. Nature 390 (6655) 70-74 (1997)

17. Linster, C., Cleland, T.A. How spike synchronization among olfactory neurons can contribute to sensory discrimination. Journal of Computational Neuroscience 10 (2) 187-193 (2001)

18. Zhang, R., et al. Boolean Chaos. Physical Review E 80 (4) (2009)

19. Rosselló, J.L., Canals, V., De Paúl, I., Bota, S., Morro, A. A simple CMOS chaotic integrated circuit. IEICE Electronics Express 5 (22) 921-926 (2008)

\section{Acknowledgements}

This work was supported in part by the Spanish Ministry of Science and Technology and in part by the Regional European Development Funds (FEDER) under EU Project TEC2011-23113

\section{Author information}

Correspondence and requests for materials should be addressed to Josep L. Rosselló (j.rossello@uib.es). Comments on this pre-print are welcome! 